\title{
POST-MINKOWSKIAN CLOSED-FORM HAMILTONIAN FOR GRAVITATING $N$-BODY SYSTEMS
}

\author{
TOMÁŠ LEDVINKA*, GERHARD SCHÄFER** and JIŘÍ BIČÁK* \\ *Institute of Theoretical Physics \\ Charles University, Prague \\ ** Theoretisch-Physikalisches Institut \\ Friedrich-Schiller-Universität, Jena
}

\begin{abstract}
The Hamiltonian for a system of relativistic bodies interacting by their gravitational field is found in the post-Minkowskian approximation, including all terms linear in the gravitational constant. It is given in a surprisingly simple closed form as a function of canonical variables describing the bodies only. The field is eliminated by solving inhomogeneous wave equations, applying transverse-traceless projections, and using the Routh functional. By including all special relativistic effects our Hamiltonian extends the results described in classical textbooks of theoretical physics. As an application, the scattering of relativistic objects is considered.
\end{abstract}

Keywords: Post-Minkowskian approximation

Post-Newtonian (PN) approximation methods in general relativity are based on the weak-field limit in which the metric is close to the Minkowski metric and the assumption that the typical velocity $v$ in a system divided by the speed of light is very small. In post-Minkowskian (PM) approximation methods only the weakness of the gravitational field is assumed but no assumption about slowness of motion is made. In the PM approximation we obtain ${ }^{1}$ the Hamiltonian for gravitationally interacting particles that includes all terms linear in gravitational constant $G$. It thus yields PN approximations to any order in $1 / c$ when terms linear in $G$ are considered; and it can also describe particles with ultrarelativistic velocities or with zero rest mass.

We use the canonical formalism of Arnowitt, Deser, and Misner (ADM ${ }^{2}$ where the independent degrees of freedom of the gravitational field are described by $h_{i j}^{T T}$, the transverse-traceless part of $h_{i j}=g_{i j}-\delta_{i j}\left(h_{i i}^{T T}=0, h_{i j, j}^{T T}=0, i, j=1,2,3\right)$, and by conjugate momenta $c^{3} /(16 \pi G) \pi^{i j T T}$. The field is generated by $N$ particles with rest masses $m_{a}$ located at $\mathbf{x}_{a}, a=1, \ldots N$, and with momenta $\mathbf{p}_{a}$. We start with the Hamiltonian ${ }^{3}$ correct up to $G^{2}$ found by the expansion of the Einstein equations (the energy and momentum constraints) in powers of $G$ and by the use of suitable regularization procedures. When we consider only terms linear in $G$ and put $c=1$ this Hamiltonian reads

$$
\begin{aligned}
H_{\text {lin }}= & \sum_{a} \bar{m}_{a}-\frac{1}{2} G \sum_{a, b \neq a} \frac{\bar{m}_{a} \bar{m}_{b}}{r_{a b}}\left(1+\frac{p_{a}^{2}}{\bar{m}_{a}^{2}}+\frac{p_{b}^{2}}{\bar{m}_{b}^{2}}\right) \\
& +\frac{1}{4} G \sum_{a, b \neq a} \frac{1}{r_{a b}}\left(7 \mathbf{p}_{a} \cdot \mathbf{p}_{b}+\left(\mathbf{p}_{a} \cdot \mathbf{n}_{a b}\right)\left(\mathbf{p}_{b} \cdot \mathbf{n}_{a b}\right)\right)-\frac{1}{2} \sum_{a} \frac{p_{a i} p_{a j}}{\bar{m}_{a}} h_{i j}^{T T}\left(\mathbf{x}=\mathbf{x}_{a}\right) \\
& +\frac{1}{16 \pi G} \int d^{3} x\left(\frac{1}{4} h_{i j, k}^{T T} h_{i j, k}^{T T}+\pi^{i j T T} \pi^{i j T T}\right),
\end{aligned}
$$


where $\bar{m}_{a}=\left(m_{a}^{2}+\mathbf{p}_{a}^{2}\right)^{\frac{1}{2}}, \mathbf{n}_{a b} r_{a b}=\mathbf{x}_{a}-\mathbf{x}_{b},\left|\mathbf{n}_{a b}\right|=1$. The equations of motion for particles are standard Hamilton equations, the Hamilton equations for the field read

$$
\dot{\pi}^{i j T T}=-16 \pi G \delta_{k l}^{T T i j} \frac{\delta H}{\delta h_{k l}^{T T}}, \quad \dot{h}_{i j}^{T T}=16 \pi G \delta_{i j}^{T T k l} \frac{\delta H}{\delta \pi^{k l T T}}
$$

here the variational derivatives and the TT-projection operator $\delta_{k l}^{T T i j}=$ $\frac{1}{2}\left(\Delta_{i k} \Delta_{j l}+\Delta_{i l} \Delta_{j k}-\Delta_{i j} \Delta_{k l}\right) \Delta^{-2}, \Delta_{i j}=\delta_{i j} \Delta-\partial_{i} \partial_{j}$, appear. These equations imply the equations for the gravitational field in the first PM approximation to be the wave equations with point-like sources $\sim \delta^{(3)}\left(\mathbf{x}-\mathbf{x}_{a}\right)$. Since both the field and the accelerations $\dot{\mathbf{p}}_{a}$ are proportional to $G$, the changes of the field due to the accelerations of particles are of the order $O\left(G^{2}\right)$. Thus, in this approximation, wave equations can be solved assuming field to be generated by unaccelerated motion of particles, i.e., it can be written as a sum of boosted static spherical fields:

$$
h_{i j}^{T T}(\mathbf{x})=\delta_{i j}^{T T k l} \sum_{b} \frac{4 G}{\bar{m}_{b}} \frac{1}{\left|\mathbf{x}-\mathbf{x}_{b}\right|} \frac{p_{b k} p_{b l}}{\sqrt{1-\dot{\mathbf{x}}_{b}^{2} \sin ^{2} \theta_{b}}},
$$

where $\mathbf{x}-\mathbf{x}_{a}=\mathbf{n}_{a}\left|\mathbf{x}-\mathbf{x}_{a}\right|$ and $\cos \theta_{a}=\mathbf{n}_{a} \cdot \dot{\mathbf{x}}_{a} /\left|\dot{\mathbf{x}}_{a}\right|$. Surprisingly, it is possible to convert the projection $\delta_{i j}^{T T k l}$ (which involves solving two Poisson equations) into an inhomogeneous linear second order ordinary differential equation and write

$$
\begin{aligned}
& h_{i j}^{T T}(\mathbf{x})=\sum_{b} \frac{G}{\left|\mathbf{x}-\mathbf{x}_{b}\right|} \frac{1}{\bar{m}_{b}} \frac{1}{y(1+y)^{2}}\left\{\left[y \mathbf{p}_{b}^{2}-\left(\mathbf{n}_{b} \cdot \mathbf{p}_{b}\right)^{2}(3 y+2)\right] \delta_{i j}\right. \\
& +2\left[1-\dot{\mathbf{x}}_{b}^{2}\left(1-2 \cos ^{2} \theta_{b}\right)\right] p_{b i} p_{b j}+\left[(2+y)\left(\mathbf{n}_{b} \cdot \mathbf{p}_{b}\right)^{2}-\left(2+3 y-2 \dot{\mathbf{x}}_{b}^{2}\right) \mathbf{p}_{b}^{2}\right] n_{b i} n_{b j} \\
& \left.+2\left(\mathbf{n}_{b} \cdot \mathbf{p}_{b}\right)\left(1-\dot{\mathbf{x}}_{b}^{2}+2 y\right)\left(n_{b i} p_{b j}+p_{b i} n_{b j}\right)\right\}+O\left(\bar{m}_{b} \dot{\mathbf{x}}_{b}-\mathbf{p}_{b}\right) G+O\left(G^{2}\right)
\end{aligned}
$$

here $y=y_{b} \equiv \sqrt{1-\dot{\mathbf{x}}_{b}^{2} \sin ^{2} \theta_{b}}$ and we anticipate $O\left(\bar{m}_{b} \dot{\mathbf{x}}_{b}-\mathbf{p}_{b}\right) \sim G$.

In the next step we use the Routh functional (see, e.g., Ref. ${ }^{4}$ )

$$
R\left(\mathbf{x}_{a}, \mathbf{p}_{a}, h_{i j}^{T T}, \dot{h}_{i j}^{T T}\right)=H-\frac{1}{16 \pi G} \int d^{3} x \pi^{T T i j} \dot{h}_{i j}^{T T}
$$

which is "the Hamiltonian for the particles but the Lagrangian for the field." Since the functional derivatives of Routhian vanish if the field equations hold, the (nonradiative) solution (3) can be substituted into the Routh functional without changing the Hamilton equations for the particles. Using the Gauss's law, an integration by parts and similar standard steps (such as dropping out total time derivatives, i.e. a canonical transformation) and the explicit substitution for $h_{i j}^{T T}\left(\mathbf{x}=\mathbf{x}_{a}\right)$ we 
get the Hamiltonian for a $N$-particle gravitating system in the PM approximation:

$$
\begin{aligned}
& H_{\operatorname{lin}}=\sum_{a} \bar{m}_{a}+\frac{1}{4} G \sum_{a, b \neq a} \frac{1}{r_{a b}}\left(7 \mathbf{p}_{a} \cdot \mathbf{p}_{b}+\left(\mathbf{p}_{a} \cdot \mathbf{n}_{a b}\right)\left(\mathbf{p}_{b} \cdot \mathbf{n}_{a b}\right)\right)-\frac{1}{2} G \sum_{a, b \neq a} \frac{\bar{m}_{a} \bar{m}_{b}}{r_{a b}} \\
& \times\left(1+\frac{p_{a}^{2}}{\bar{m}_{a}^{2}}+\frac{p_{b}^{2}}{\bar{m}_{b}^{2}}\right)-\frac{1}{4} G \sum_{a, b \neq a} \frac{1}{r_{a b}} \frac{\left(\bar{m}_{a} \bar{m}_{b}\right)^{-1}}{\left(y_{b a}+1\right)^{2} y_{b a}}\left[2 \left(2\left(\mathbf{p}_{a} \cdot \mathbf{p}_{b}\right)^{2}\left(\mathbf{p}_{b} \cdot \mathbf{n}_{b a}\right)^{2}\right.\right. \\
& \left.-2\left(\mathbf{p}_{a} \cdot \mathbf{n}_{b a}\right)\left(\mathbf{p}_{b} \cdot \mathbf{n}_{b a}\right)\left(\mathbf{p}_{a} \cdot \mathbf{p}_{b}\right) \mathbf{p}_{b}^{2}+\left(\mathbf{p}_{a} \cdot \mathbf{n}_{b a}\right)^{2} \mathbf{p}_{b}^{4}-\left(\mathbf{p}_{a} \cdot \mathbf{p}_{b}\right)^{2} \mathbf{p}_{b}^{2}\right) \frac{1}{\bar{m}_{b}^{2}}+2\left[-\mathbf{p}_{a}^{2}\left(\mathbf{p}_{b} \cdot \mathbf{n}_{b a}\right)^{2}\right. \\
& \left.+\left(\mathbf{p}_{a} \cdot \mathbf{n}_{b a}\right)^{2}\left(\mathbf{p}_{b} \cdot \mathbf{n}_{b a}\right)^{2}+2\left(\mathbf{p}_{a} \cdot \mathbf{n}_{b a}\right)\left(\mathbf{p}_{b} \cdot \mathbf{n}_{b a}\right)\left(\mathbf{p}_{a} \cdot \mathbf{p}_{b}\right)+\left(\mathbf{p}_{a} \cdot \mathbf{p}_{b}\right)^{2}-\left(\mathbf{p}_{a} \cdot \mathbf{n}_{b a}\right)^{2} \mathbf{p}_{b}^{2}\right] \\
& +\left[-3 \mathbf{p}_{a}^{2}\left(\mathbf{p}_{b} \cdot \mathbf{n}_{b a}\right)^{2}+\left(\mathbf{p}_{a} \cdot \mathbf{n}_{b a}\right)^{2}\left(\mathbf{p}_{b} \cdot \mathbf{n}_{b a}\right)^{2}+8\left(\mathbf{p}_{a} \cdot \mathbf{n}_{b a}\right)\left(\mathbf{p}_{b} \cdot \mathbf{n}_{b a}\right)\left(\mathbf{p}_{a} \cdot \mathbf{p}_{b}\right)\right. \\
& \left.\left.+\mathbf{p}_{a}^{2} \mathbf{p}_{b}^{2}-3\left(\mathbf{p}_{a} \cdot \mathbf{n}_{b a}\right)^{2} \mathbf{p}_{b}^{2}\right] y_{b a}\right], \quad \frac{1}{\bar{m}_{b}} \sqrt{m_{b}^{2}+\left(\mathbf{n}_{b a} \cdot \mathbf{p}_{b}\right)^{2}}
\end{aligned}
$$

Since the PM approximation can describe ultrarelativistic or zero-rest-mass particles, we calculated gravitational scattering of two such particles using the Hamiltonian (66). If perpendicular separation $\mathbf{b}$ of trajectories $(|\mathbf{b}|$ is the impact parameter) in the center-of-mass system $\left(\mathbf{p}_{1}=-\mathbf{p}_{2} \equiv \mathbf{p}\right)$ is used, $\mathbf{p} \cdot \mathbf{b}=0$, we find, after evaluating a few simple integrals, that the exchanged momentum in the system is given by

$$
\Delta \mathbf{p}=-2 \frac{\mathbf{b}}{\mathbf{b}^{2}} \frac{G}{|\mathbf{p}|} \frac{\bar{m}_{1}^{2} \bar{m}_{2}^{2}}{\bar{m}_{1}+\bar{m}_{2}}\left[1+\left(\frac{1}{\bar{m}_{1}^{2}}+\frac{1}{\bar{m}_{2}^{2}}+\frac{4}{\bar{m}_{1} \bar{m}_{2}}\right) \mathbf{p}^{2}+\frac{\mathbf{p}^{4}}{\bar{m}_{1}^{2} \bar{m}_{2}^{2}}\right] .
$$

The quartic term is all that remains from the field part $h_{i j}^{T T}$ in agreement with Westpfah $\sqrt{5}$ who used a very different approach.

The Hamiltonian (6) can also describe a binary system with one massless and one massive particle orbiting around each other. This is not obvious: the second, fourth or even sixth-order PM approximation would not be able to describe massless test particles orbiting around a Schwarzschild black hole.

We acknowledge the support from SFB/TR7 in Jena, from the Grant GAČR 202/09/0772 of the Czech Republic, and of Grants No LC 06014 and the MSM 0021620860 of Ministry of Education.

\section{References}

1. T. Ledvinka, G. Schäfer and J. Bičák, Phys. Rev. Lett. 100, 251101 (2008).

2. R. Arnowitt, S. Deser and C. W. Misner, The dynamics of general relativity, in Gravitation: An Introduction to Current Research, ed. L. Witten (Wiley, New York, 1962)

3. G. Schäfer, Gen. Relativ. Gravit. 18, 255 (1986).

4. P. Jaranowski and G. Schäfer, Phys. Rev. D 57, 7274 (1998), Erratum-ibid. D 63, 029902 (2001).

5. K. Westpfahl, Fortschr. der Physik 33, 417 (1985). 